\title{
A convolutional neural network for the quality control of MRI defacing
}

\author{
Daniel J. Delbarre ${ }^{\mathrm{a}, *}$, Luis Santos ${ }^{\mathrm{a}}$, Habib Ganjgahi ${ }^{\mathrm{b}}$, Neil Horner $^{\mathrm{a}}$, Aaron McCoy ${ }^{\mathrm{a}}$, Henrik \\ Westerberg $^{\mathrm{a}}$, Dieter A. Häring ${ }^{\mathrm{c}}$, Thomas E. Nichols ${ }^{\mathrm{d}, \mathrm{e}}$, Ann-Marie Mallon ${ }^{\mathrm{a}}$ \\ ${ }^{a}$ MRC Harwell Institute, Harwell Campus, Oxfordshire, OX11 oRD, United Kingdom \\ ${ }^{b}$ Department of Statistics, University of Oxford, 24-29 St Giles', Oxford, OX1 3LB, Oxford, United Kingdom \\ ${ }^{c}$ Novartis Pharma AG, Basel, Switzerland \\ ${ }^{d}$ Big Data Institute, Li Ka Shing Centre for Health Information and Discovery, Nuffield Department of Population \\ Health, University of Oxford, Old Road Campus, Oxford, OX3 $7 L F$, United Kingdom \\ ${ }^{e}$ Wellcome Centre for Integrative Neuroimaging, FMRIB, Nuffield Department of Clinical Neurosciences, University of \\ Oxford, Oxford, OX3 9DU, United Kingdom
}

\begin{abstract}
Large scale neuroimaging datasets present unique challenges for automated processing pipelines. Motivated by a large-scale clinical trials dataset of Multiple Sclerosis (MS) with over 235,000 magnetic resonance imaging (MRI) scans, we consider the challenge of defacing - anonymisation to remove identifying features on the face and the ears. The defacing process must undergo quality control (QC) checks to ensure that the facial features have been adequately anonymised and that the brain tissue is left completely intact. Visual QC checks - particularly on a project of this scale - are time-consuming and can cause delays in preparing data for research. In this study, we have developed a convolutional neural network (CNN) that can assist with the QC of MRI defacing. Our CNN is able to distinguish between scans that are correctly defaced, and three sub-types of failures with high test accuracy (77\%). Through applying visualisation techniques, we are able to verify that the CNN uses the same anatomical features as human scorers when selecting classifications. Due to the sensitive nature of the data, strict thresholds are applied so that only classifications with high confidence are accepted, and scans that are passed by the CNN undergo a time-efficient verification check. Integration of the network into the anonymisation pipeline has led to nearly half of all scans being classified by the CNN, resulting in a considerable reduction in the amount of time needed for manual QC checks, while maintaining high QC standards to protect patient identities.
\end{abstract}

Keywords: magnetic resonance imaging, defacing, quality control, neural network, anonymisation

\section{Introduction}

A collaboration between Novartis and the University of Oxford's Big Data Institute (BDI) has been established to improve drug development and healthcare through the application of artificial intelligence and advanced analytics. Large, multidimensional datasets - consisting of clinical, imaging and omics 5 data - are integrated and analysed to improve patient prognoses and identify early predictors of disease. A dedicated research informatics framework has been developed that allows this data to be captured, anonymised, explored and integrated into databases (Mallon et al., 2021). Currently, the collaboration

\footnotetext{
* Corresponding author

Email address: d.delbarre@har.mrc.ac.uk (Daniel J. Delbarre)
} 
focuses on two main therapeutic areas: Multiple Sclerosis (MS), and autoimmune diseases treated with the interleukin (IL)-17A antibody secukinumab (IL17). Data from over 50,000 patients is available across both projects, and the MS project will utilise both clinical and brain magnetic resonance imaging (MRI) data - a key part of the MS project, with over 235,000 scans from over 12,000 unique subjects. Many of the subjects have longitudinal MRI data over several years and corresponding clinical information, enabling the progression of disease to be studied (Dahlke et al., 2021).

Before the MRI data can be used for research purposes it is necessary to homogenise the data to a single format, and to anonymise all confidential patient data, including metadata and any facial features that are present in the image data. While MRI metadata (stored within DICOM tags in raw MRI data) can be readily anonymised, through deletion or modification of selected tags, the anonymisation of the image data itself is more complex. Two commonly used approaches are skull stripping and defacing. Skull stripping involves removing all non-brain tissue from a scan, and can be implemented using a number of methods (e.g. Ségonne et al., 2004; Shattuck et al., 2001; Smith, 2002). However, skull-stripping methods often require considerable fine-tuning when applied to large datasets containing scans of variable image quality (Fennema-Notestine et al., 2006), and additionally, skull-stripped images can sometimes be unsuitable when similar algorithms are required for processing images downstream of de-identification work (Schimke et al., 2011). Defacing techniques retain non-brain tissue and can be implemented through shearing off parts of the face (e.g. Schimke and Hale, 2011), blurring the outer surface of the face (Milchenko and Marcus, 2013) and selectively removing areas of the face that contain identifiable facial features (e.g. Bischoff-Grethe et al., 2007; Alfaro-Almagro et al., 2018; Gulban et al., 2019).

Quality control (QC) processes are commonly employed when working with MRI data to ensure that the data is suitable for downstream analysis, and that artefacts in the data will not introduce bias into analyses (Reuter et al., 2015). Furthermore, the quality of defacing may also need to be QC checked (e.g. Marcus et al., 2013) to ensure that not only are any identifying facial features correctly removed from the scan, but also to ensure that the brain anatomy has not been damaged. The choice of defacing software is important as the successful application can be variable, particularly when the data has been acquired at different sites, with different acquisition parameters (Theyers et al., 2021). Additionally, the choice of software can impact the results of downstream analyses (Bhalerao et al., 2021; Schwarz et al., 2021). If high standards of QC checks are not employed, then there is potential risk of patients being identified through photographic visual comparisons (Prior et al., 2009), facial recognition software (Mazura et al., 2012; Schwarz et al., 2019, 2021), and the facial reconstruction of inadequately defaced MRI scans (Abramian and Eklund, 2019; Schwarz et al., 2021). Many defacing methods have been developed on high resolution, research quality scans. In this collaboration, which utilises clinical MRI data, the scans are typically lower resolution, were captured from a large number of sites, and due to the longitudinal nature of the data, some scans were acquired over 15 years ago. Therefore, due to the potentially high levels of variation in the quality of the data, there is greater potential for variation in the successful application of defacing to these scans. As a consequence of this, thorough QC checks are necessary to ensure data is correctly anonymised.

While visual QC checks are commonly employed to assess the quality of MRI scans, when undertaking 
projects containing tens of thousands, or in the case of this project hundreds of thousands of scans, these manual checks become less feasible. The time-consuming nature of the checks can cause considerable delays between receiving data and having the data research-ready. Furthermore, those undertaking the QC may become fatigued and more likely to make errors. Citizen scientists can be used to assist with visually QC checking MRI data (e.g. Keshavan et al., 2019), but this is not suitable when anonymisation checks are being undertaken. Automated methods have been developed to assist with the QC of MRI data, utilising methods including univariate classifiers (Mortamet et al., 2009), support vector machines (SVMs; Pizarro et al., 2016), random forests (Klapwijk et al., 2019), neural networks (Litjens et al., 2017), or a combination of classifiers (Alfaro-Almagro et al., 2018; Esteban et al., 2017). However, existing automated QC models are focused on QC checking image quality or analysis output, and currently no models exist for assisting with the QC of MRI defacing.

In this work, we developed a convolutional neural network (CNN) to assist with the QC checking of defaced MRI images. Our network was developed using 24,000 pre-classified renders of defaced scans. Following development of the CNN, we evaluate model performance to select strict probability thresholds that convey high levels of accuracy. We also describe the integration of the CNN into a pre-existing image anonymisation pipeline, including the adoption of time-efficient verification checks to protect patient anonymity. Furthermore, we evaluate the implications of implementing the CNN with regard to time saved compared to fully manual QC checks, and discuss the potential for the adoption of machine learning approaches to improve efficiency of MRI pre-processing.

\section{Material and methods}

\subsection{MRI data}

MRI data was available from 24 Novartis clinical studies, with the entire dataset containing data from over 12,000 subjects and over 235,000 MRI scans. The majority of scan sessions contained T1-weighted (with and without Gadolinium contrast enhancement), T2-weighted and proton density modalities. Some subjects also had T1-weighted 3D acquisitions, diffusion-weighted, FLAIR, and magnetisation transfer scans. MRI scans were of variable quality as they were captured from over 1,000 unique MRI scanners, with some scans captured over 15 years prior to the start of the collaboration. The majority of scans had a 256 × 256 or $192 \times 256$ acquisition matrix, with 46 or 60 slices.

\subsection{MRI defacing pipeline prior to $C N N$ development}

MRI data was initially transferred to a dedicated, secure anonymisation environment for defacing and the anonymisation of any remaining confidential patient data. The anonymisation environment was only accessible to a very small team of dedicated scientists not otherwise involved in the research. A bespoke pipeline (Fig. 1) was built to handle the processing of this data into a consistent format, ready for downstream research. Data was initially converted to the Neuroimaging Informatics Technology Initiative (NIfTI) format using the DICOM conversion software HeuDiConv (Halchenko et al., 2019), and structured using the Brain Imaging Data Structure standard (BIDS; Gorgolewski et al., 2016) - 


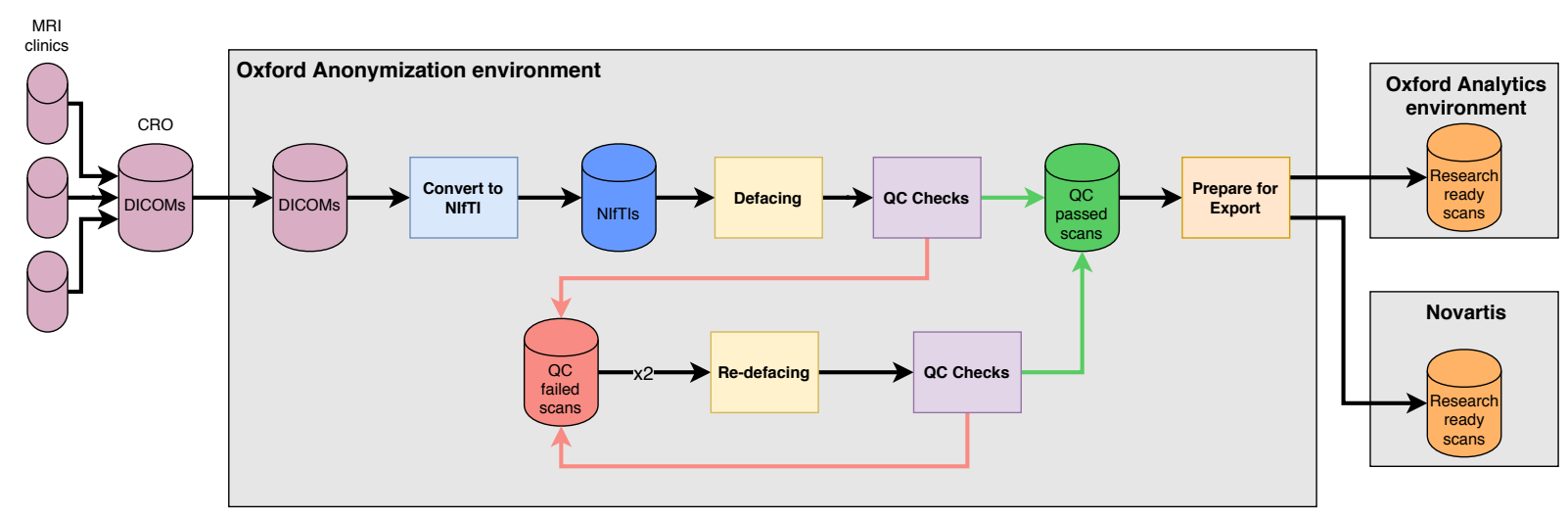

Figure 1: Flowchart showing the anonymisation pipeline for MRI data prior to development of the CNN.

a standard for brain MRI datasets, that is widely used within the neuroimaging research community. Anonymised metadata was preserved in a JSON file that accompanied each NIfTI file.

After conversion to NIfTI format, each scan was defaced. During this process, identifying facial features, specifically the ears, eyes, nose and mouth, are removed from each scan. Defacing was implemented using the fsl_deface software (Alfaro-Almagro et al., 2018), which is known to perform well at preventing re-identification and minimise interference with downstream analysis methods when compared to other defacing software (Schwarz et al., 2021). Following being defaced, all scans were QC checked to ensure that the defacing had been applied correctly. Scans were classified as one of four categories: pass (scan defaced correctly), deep (defacing went too deep and encroached on the brain), shallow (defacing did not go deep enough and facial features were still visible), failure (broad category for complete registration failures and scans containing unfixable errors). Prior to the development of the neural network, all QC checks were carried out manually, using a HTML page generated by a Python script. Each of the scans were visualised as two PNG images of 3D renders of the scan (left and right side) which were generated using the fsl_gen_3D script (Alfaro-Almagro et al., 2018). Using the HTML page, each scan was classified by clicking the button that corresponds to one of the above categories (Fig. 2).

After the initial QC checks were completed, scans which did not pass were re-defaced using custom parameters that were selected by considering the type of scan and the previous QC classification(s). Following each round of the re-defacing, scans were once again QC checked using the same protocol as with the initial defacing. Up to two rounds of re-defacing were undertaken to ensure that as many scans as possible passed the defacing stage; on average $96 \%$ of scans were successfully defaced. All anonymised NIfTI scans, and accompanying JSON metadata files, that passed the QC checks were then prepared for export to a separate, analysis-focused environment at the BDI and also back to Novartis, ready to be included in downstream research and analysis.

\subsection{CNN: Data selection and pre-processing}

As over 100,000 scans had been manually QC checked prior to the development of the neural network, a large quantity of labelled scan data was available for use in developing the CNN. However, not all of this data could be used in network development for two reasons. Firstly, as the majority of prior manual QC classifications were for scans classified as 'pass' (75\%) and to a lesser extent 'shallow' (15\%), there 

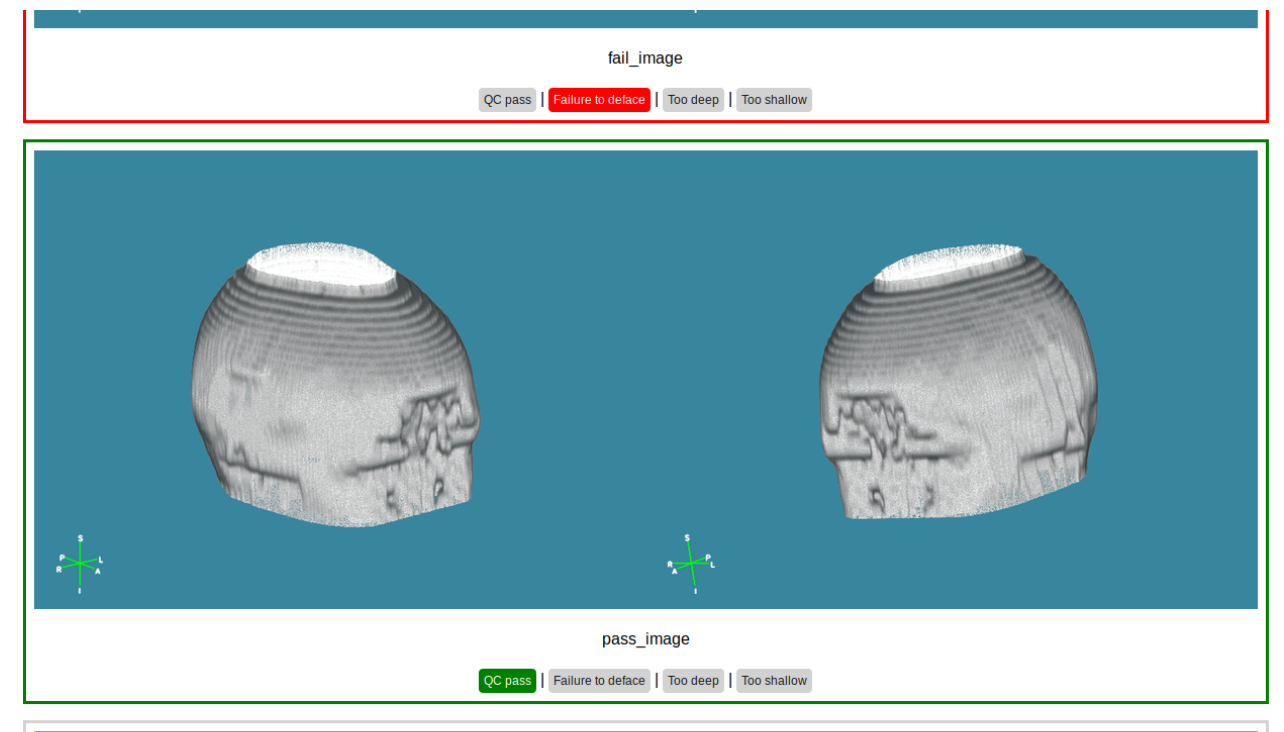

Figure 2: Usage of the html page when undertaking manual QC checks. Note that the renders in this figure are generated from a sample scan from I Do Imaging (2017).

was considerably less 'deep' (6\%) and 'failure' (4\%) classification data, so in order to keep the proportion of data from each class balanced (at least initially; see below) a smaller susbset of the available scans were used. Secondly, as most subjects usually have multiple scans (usually $8+$, but up to 60) and some of these scans had been re-defaced and therefore QC checked multiple times, it was preferable not to use all available scans to reduce the chance of the CNN overfitting to the anatomy of subjects appearing in the dataset multiple times.

Initially, 16,000 scans were used to develop the model, with 4,000 scans per class, which were split 60:20:20 between training, validation and test sets. Due to the initial models performing better on the 'deep' and 'failure' classes, the proportion of 'shallow' and 'pass' scans in the dataset was increased to 8,000 scans per class, giving 24,000 scans in total. Scans for each class were randomly selected from those available, and included scans from multiple clinical studies, all available scan modalities, and they were of varying image quality.

A single 2D image was used as the input for the CNN. Each image was a composite of the two renders used in the manual QC checks, which were cropped to remove parts of the background that did not contain parts of the anatomy in any scans, and then joined side-by-side to create a $550 \mathrm{x} 900$ (height $\mathrm{x}$ width) pixel image. The rationale for using the $2 \mathrm{D}$ renders over the $3 \mathrm{D}$ scans was as follows: the average NIfTI scan in this project contains over 3 million voxels, most of which is not data that is applicable to assessing the quality of the defacing (and would be computationally expensive to process in its entirety). In addition, while image registration could be used to extract the head/face region (and downsample the $3 \mathrm{D}$ data), defacing failures are often the result of poor image registration. Therefore, by using 2D images of the renders, visual information that is pertinent to the effectiveness of the defacing is retained in a more compact format, and the style of the renders is consistent regardless of image quality. 


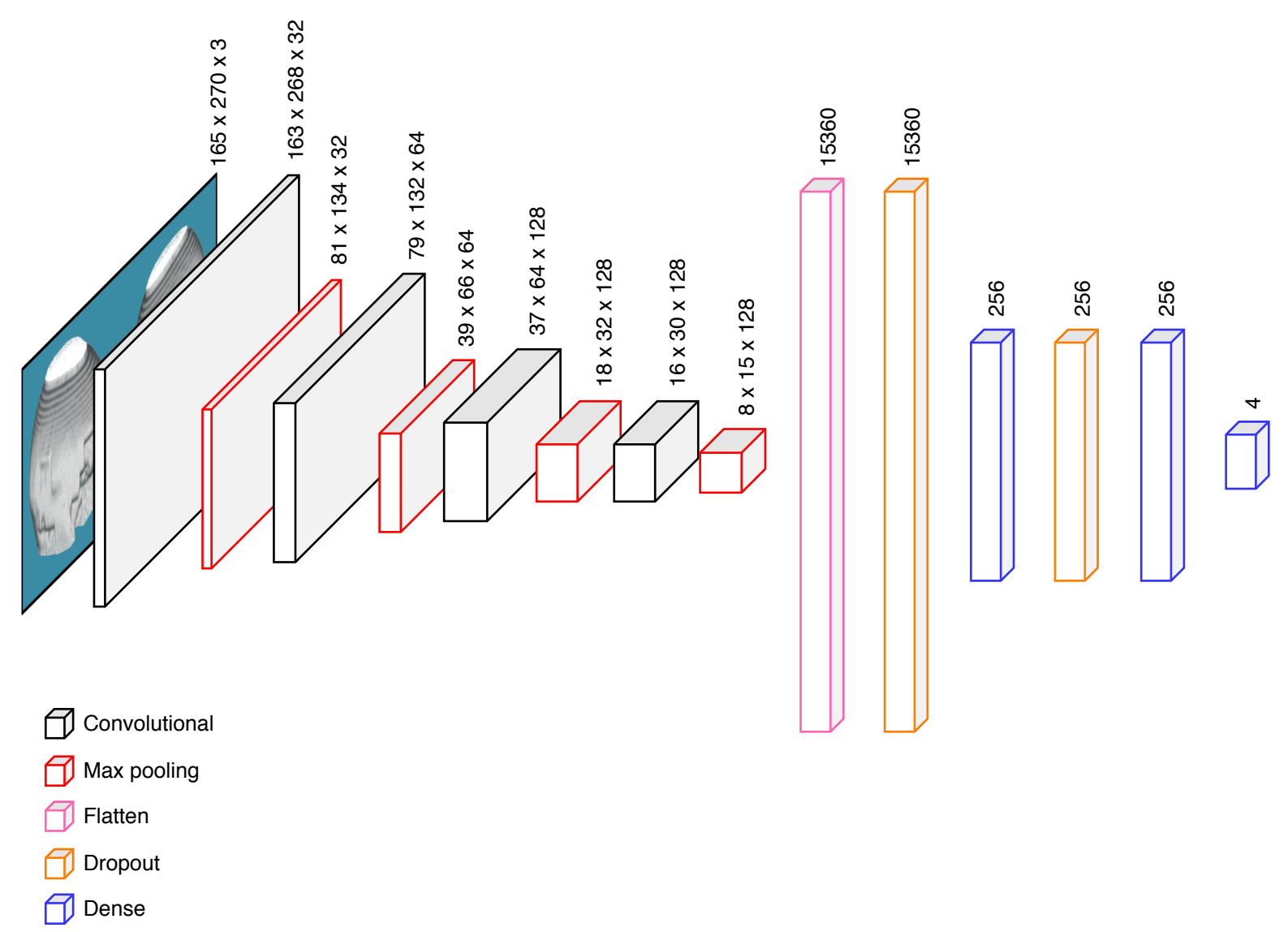

Figure 3: Network diagram of the CNN.

\subsection{CNN: Network development}

The CNN was developed using the 'keras' library (Allaire and Chollet, 2018) for R (R Core Team, 2018). Images were input to the network as $165 \times 270 \times 3$ (height $\mathrm{x}$ width $\mathrm{x}$ depth) tensors with values scaled between 0-1. The image order was shuffled and image augmentation (zoom, horizontal flipping) was used on the training data set to reduce overfitting; augmentation was not applied to validation or test data. The base of the network (Fig. 3) contained four convolutional layers, each using a rectified linear unit (ReLU) activation function and a kernel size of $3 \times 3$. Each convolutional layer was followed by a max pooling layer to downsample the data. After flattening, two dropout and three densely connected layers were interspersed. Both dropout layers had a dropout rate of 0.2. The first two dense layers used ReLU activation, with the final dense layer using softmax activation with an output size of four - one for each of the four classes. The network was optimised using a root mean square optimiser (RMS) with the learning rate set to 0.00008 , and categorical cross-entropy was used as the loss function. The network was trained for 100 epochs, with 576 steps and a batch size of 25, so that each epoch contained all 14,400 training images. During each epoch the model was run on the validation set, with 192 steps and a batch size of 25. A callback was used to save the best performing model during training, using minimised validation loss as the criterion. 
bioRxiv preprint doi: https://doi.org/10.1101/2021.06.24.449613; this version posted June 25,2021 . The copyright holder for this preprint (which was not certified by peer review) is the author/funder, who has granted bioRxiv a license to display the preprint in perpetuity. It is made available under aCC-BY-NC-ND 4.0 International license.

In developing the network, a number of different architectures were tested before selecting the final model. This included testing a transfer learning approach where a new densely connected classifier was added to a convolutional base from a network trained on the ImageNet database. Additional networks were built from scratch and modifications were made in an iterative manner to evaluate model performance. Modifications included adjusting the number of layers, numbers of neurons, types of layers, the optimiser, learning rates, input sizes and the use of image augmentation. Modifications were evaluated by comparing the validation loss and validation accuracy for the best performing model from each network.

The assignment of classifications was visualised using Gradient-weighted Class Activation Mapping (Grad-CAM; Selvaraju et al., 2017). This approach allowed for the activation of a class for an input image to be visualised, which shows what features of the MRI renders the CNN is using to assign classifications. The Grad-CAM method was implemented in R using the approach of Chollet and Allaire (2018), where a semi-transparent heatmap of the predicted class activations from the final convolutional layer is superimposed on top of the input image. For illustrative purposes, a publicly-available sample T2w MRI dataset (I Do Imaging, 2017) was used for the images included here; the parameters of the defacing script were modified to produce an example of each of the four classes.

Before the network was incorporated into the defacing pipeline, cut-off probability thresholds were selected so that only classifications with high confidence would be accepted. This was to ensure that 1) patient anonymity is preserved by not allowing inadequately defaced scans to be marked as 'pass', and 2) so that incorrectly defaced scans were assigned to the correct class and that the appropriate re-defacing parameters could be applied downstream. Cut-off probability thresholds were selected using metrics including sensitivity, specificity, and volume of data that would be classified at different thresholds (between $0.25-1.00$ at 0.05 increments) for each class on the test dataset. Classifications were assigned based on the class with the highest probability. As the classifications for images that did not reach a probability threshold would not be used (see below), the above metrics only included data that would be classified.

Multi-class receiver operating characteristic (ROC) curves, and the corresponding area under the curves (AUC) were calculated using the method of Hand and Till (2001), as implemented in the pROC R package (Robin et al., 2011).

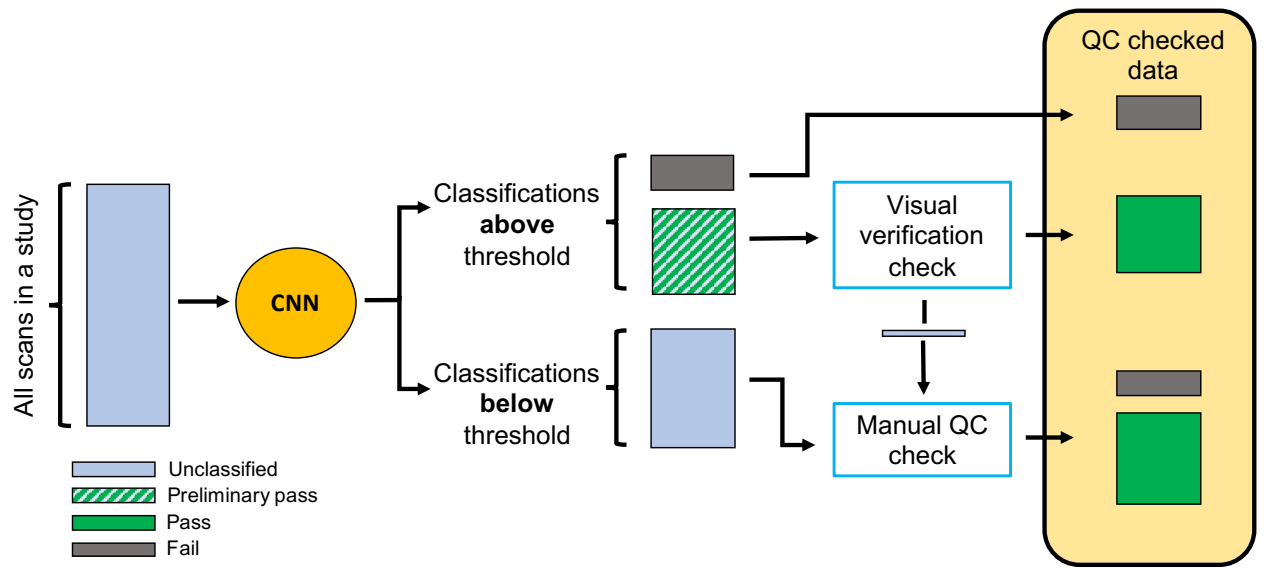

Figure 4: Flowchart showing how the CNN has been integrated into the anonymisation pipeline. 


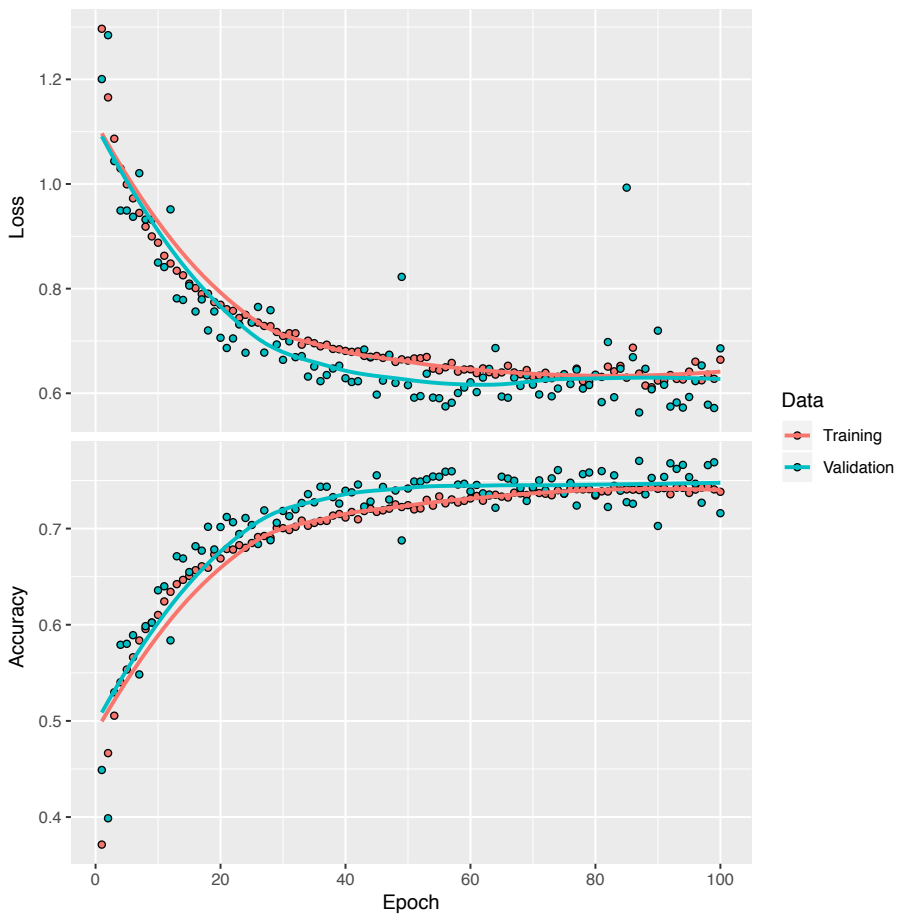

Figure 5: Loss and accuracy of the training and validation datasets during each epoch of training the CNN.

\subsection{Integrating the $C N N$ into the defacing pipeline}

The CNN was integrated into the defacing pipeline (Fig. 4), and was used at the initial defacing and the two re-defacing stages. Following the defacing of all scans in a study, a composite image of the 2 renders was generated to match the format of the images used in training the network. Classifications for the three non-pass categories were accepted when they exceeded the cut-off probability threshold for a class. Pass classifications that surpassed the pass cut-off threshold were preliminarily accepted, pending a visual verification check before being fully accepted. The visual verification check is a quicker version of the full manual QC check, where the images are displayed in a gallery view that can be swiftly browsed and only those images where the pass classification is not agreed with are flagged. Any scans that were flagged during the verification check, and the remainder of the scans that did not reach any of the cut-off thresholds, were then QC checked using the original manual method.

After the CNN was incorporated into the pipeline, its impact was assessed by evaluating the number of scans that were processed, the classifications that were assigned, and the time taken to undertake QC checks. The efficiency savings by using the CNN, compared to fully manual QC checks, was determined by calculating the time savings for a hypothetical study containing 10,000 scans. The performance of the CNN (e.g. proportions of scans classified in the four classes, the proportion of CNN assigned passes accepted during verification checks) was based on the performance of the CNN on data processed by it so far. The time taken to perform the manual and verification checks was determined by calculating the average time it takes to check a single scan in a batch of 250 scans. 
a)

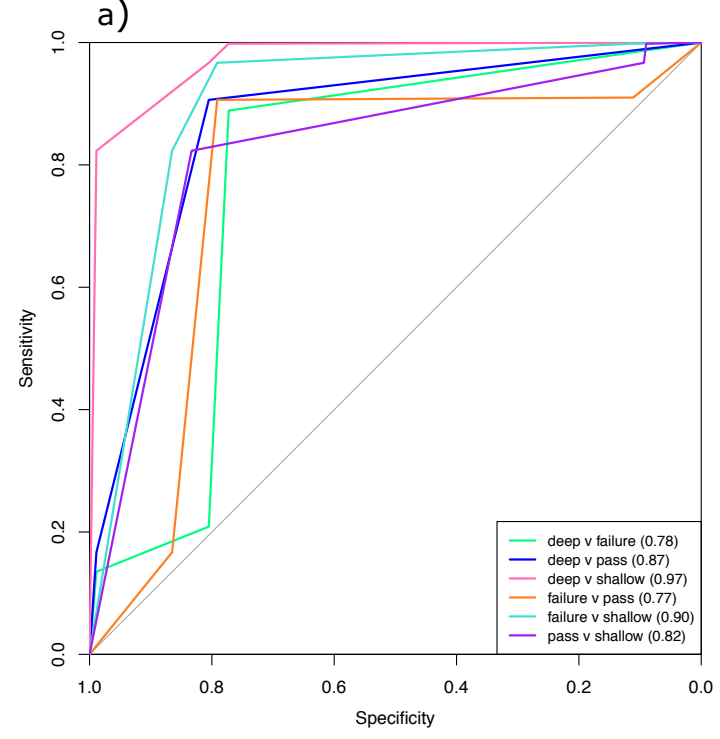

b)

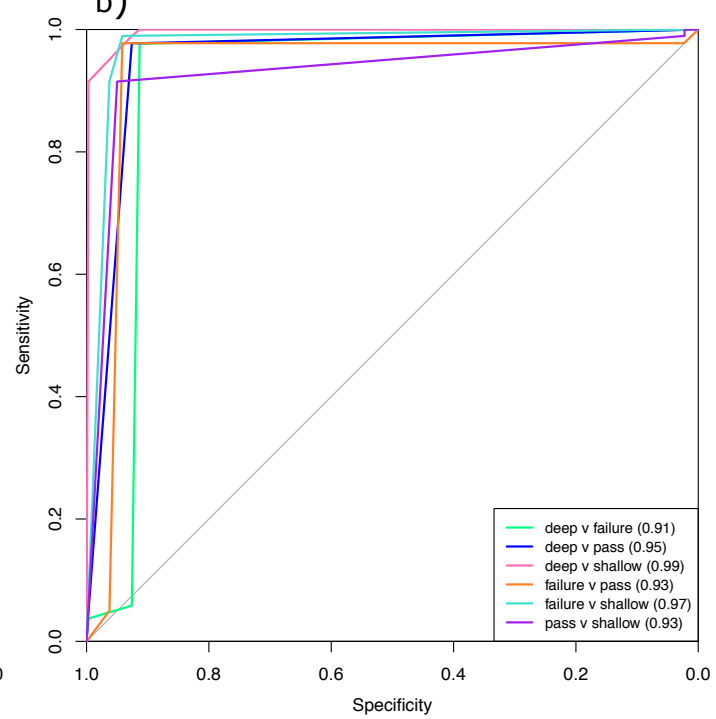

Figure 6: Multi-class ROC curves when a) all classifications are accepted, and b) when the thresholds are applied. Each curve represents one pair-wise comparison. The AUC is shown in the legend for each comparison.

\section{Results}

\subsection{Network performance}

During training of the final network, both training and validation loss steadily decreased over 60 epochs, after which there were smaller improvements up until around 90 epochs when the loss plateaued high levels of activation above the forehead. With a complete defacing failure, where behind the face has been defaced and the defacing has gone quite deep into the sides of the head (Fig. 7b), there is very 


\section{(a) Pass - 0.9497}

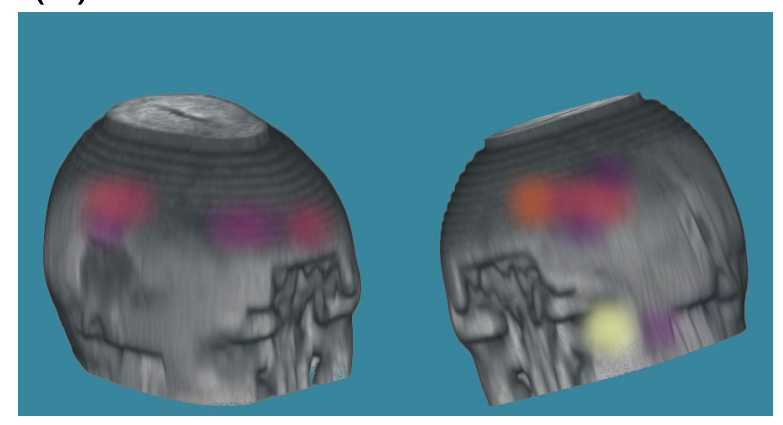

\section{(c) Deep - 0.9991}

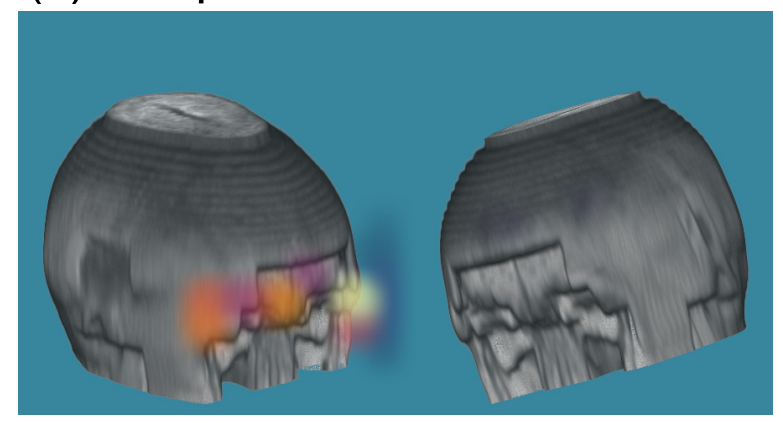

(b) Failure - 0.9999

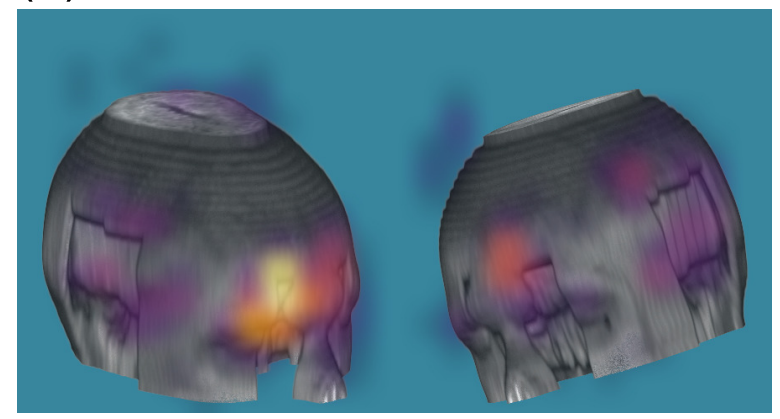

(d) Shallow - 0.9690

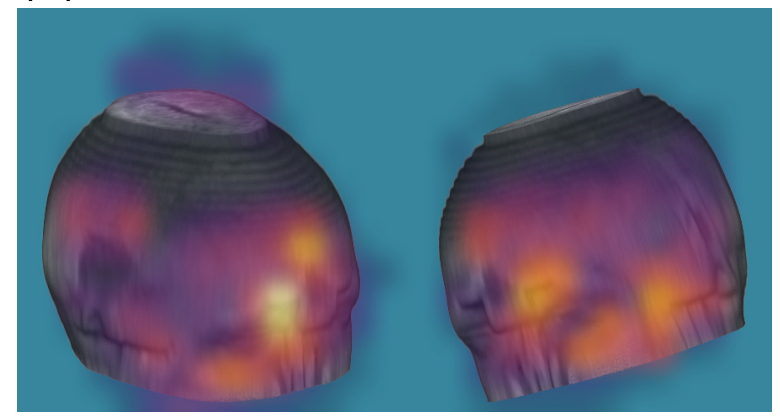

Figure 7: Grad-CAM heatmaps for representative scans of each of the four classifications, a) pass, b) registration failure, c) deep, and d) shallow. The CNN probability that each image belongs to the class is shown above each image. Each image was produced from a sample scan from I Do Imaging (2017) with the defacing parameters modified to produce different outcomes.

strong activation around a hole in the forehead. Other areas of the head where the defacing has not been adequately applied are also activated. In a scan where the defacing has gone very deep and intersected the brain (Fig. 7c), there is strong activation at the front of the brain and around the strong angular lines where the mask has cut through the sides of the front of the head. Interestingly, only one render shows any activation. The 'shallow' scan, in which the eyes are still visible (Fig. 7d), has very strong activation around the orbits, and high levels around the whole face. In particular the ' $L$ '-shaped cuts where the defacing mask has started to cut into the area of the brows, and below the eyes show strong activation.

The CNN is able to classify 1,000 images in 51 seconds (0.051 seconds per image), including the production of CSV files recording the classifications, and moving images to sub-directories based on assigned classes. When the number of images is increased to 10,000 the processing time decreases to 0.048 seconds per image (475 seconds in total).

\subsection{Probability threshold selection}

When the probability thresholds are less strict, the sensitivity and specificity decline in a similar pattern for the 'pass', 'deep' and 'shallow' classes although the specificity remains more consistent for the 'deep' class (Fig. 8). While there is a much greater decline in the sensitivity for the 'failure' class compared to the other three classes, the specificity remains very high $(>0.97)$ regardless of the threshold. When strict thresholds (0.95) are applied, the proportion of data which surpasses the selected thresholds is low 

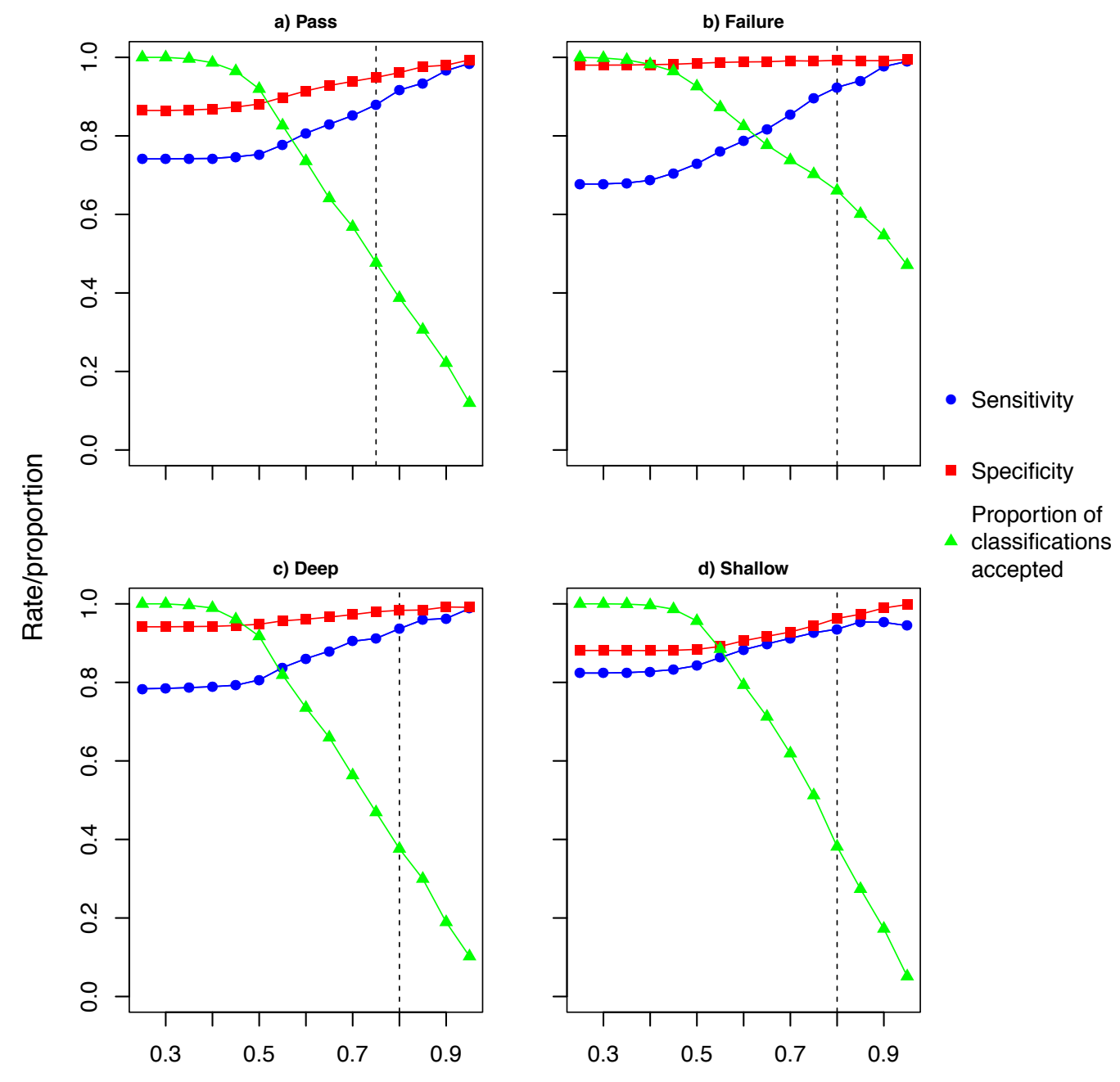

Probability threshold

Figure 8: Plots showing how model performance on the test set varies when the probability threshold of accepted classifications is modified. Each class is shown separately: a) pass, b) registration failure, c) deep, and d) shallow. Sensitivity, specificity and the proportion of classifications that would be accepted are shown for each class. Vertical dashed lines show the thresholds that were used in the final model.

for all classes $(<13 \%)$ except the 'failure' class where it is $46 \%$. After examining the overall performance of the network, a global probability threshold of 0.8 was selected for all classes, with the exception of the 'pass' class. As scans assigned the 'pass' classification would undergo a visual verification check a slightly lower threshold of 0.75 was selected. Using multi-class ROC curves (Fig. 6b), the AUCs from all comparisons are $\geq 0.91$, with most comparisons having AUCs $\geq 0.93$. With the selected thresholds applied, $45 \%$ of data in the test set would be classified by the CNN, and $92 \%$ of these predictions match the labelled classes.

\subsection{Integrating the $C N N$ into the defacing pipeline}

Since the CNN was integrated into the defacing pipeline, over 76,000 scans have been processed (Fig. 9 ), of which nearly $53 \%$ were from scans that had been through initial defacing, and the remaining $47 \%$ had been through re-defacing. The CNN classified $30 \%$ of scans as passed, $12 \%$ as shallow, $2 \%$ as 
bioRxiv preprint doi: https://doi.org/10.1101/2021.06.24.449613; this version posted June 25, 2021. The copyright holder for this preprint (which was not certified by peer review) is the author/funder, who has granted bioRxiv a license to display the preprint in perpetuity. It is made available under aCC-BY-NC-ND 4.0 International license.

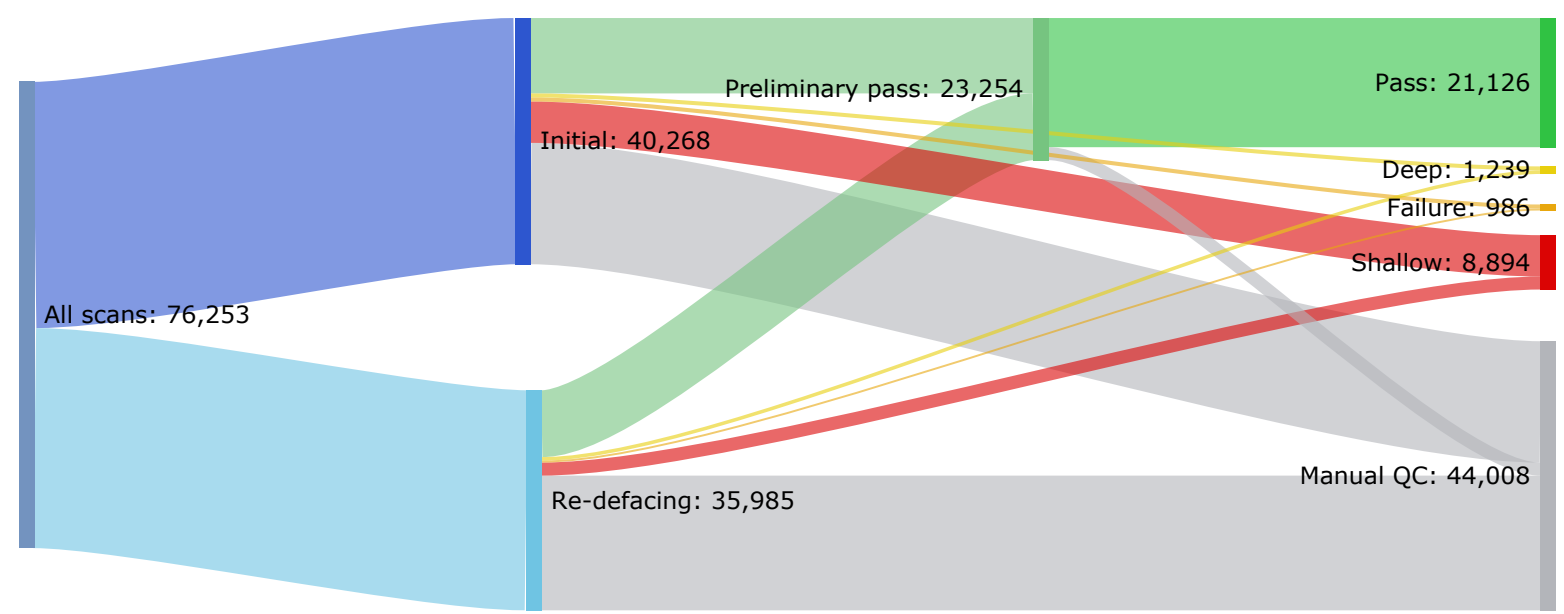

Figure 9: Sankey diagram showing scans that have been QC checked with the CNN incorporated into the anonymisation pipeline. Scans are split into those from initial defacing and re-defacing QC.

deep, and $1 \%$ as registration failures; the remaining scans were flagged for manual QC checks as they did not surpass the applied probability thresholds. Of the scans assigned 'pass', $91 \%$ and $90 \%$ of these classifications were accepted following the visual verification checks in the initial and re-defacing stages respectively. Overall, $45 \%$ of the classifications generated by the CNN were accepted, however a larger proportion of the classifications (51\%) were accepted for scans that were QC checked following initial defacing, and a much smaller proportion of classifications (39\%) were accepted for scans that had been through re-defacing. Of the total proportion of scans classified by the CNN, $60 \%$ of scans were classified as pass during initial defacing, but this was much greater during re-defacing, where $78 \%$ of scans were classified as 'pass'. This seems to be the result of a considerably smaller proportion of scans being assigned 'shallow' during re-defacing, due to the success of the revised defacing parameters that are applied during this stage.

Incorporation of the $\mathrm{CNN}$ into the pipeline has allowed for a reduction in the amount of time spent on manual QC checks. Using summary data on scans that have been processed so far, it is possible to compute the time that is saved on an average study containing 10,000 scans (Fig. 10). Typically, $31 \%$ of scans in a study will need re-defacing, and $12 \%$ of the original scans will need a second round of re-defacing. On average, it takes a human scorer 3.8 seconds to visually QC check a scan, which includes time spent waiting for the image to load on the HTML page, looking at the image, and marking the classification. The visual verification checks are much quicker, taking 0.8 seconds per scan on average. Therefore, an average study with 10,000 scans would take 15.1 hours to manually QC check. With the addition of the CNN to the pipeline the time needed for manual and verification checks during initial defacing would be nearly halved to 6.1 hours (from 10.6 hours). During re-defacing, the time savings are less due to the CNN not performing as well on re-defaced scans (i.e. these scans are more commonly borderline between classes, or the subject's anatomy or the scan quality makes defacing difficult). Verification and manual QC checks would take 2.5 hours during the first round and 1.2 hours during the second round of re-defacing when using the CNN. Prior to incorporation of the CNN the checks would have taken 3.3 and 1.3 hours during the first and second rounds. In total, QC checks with the CNN incorporated into the 


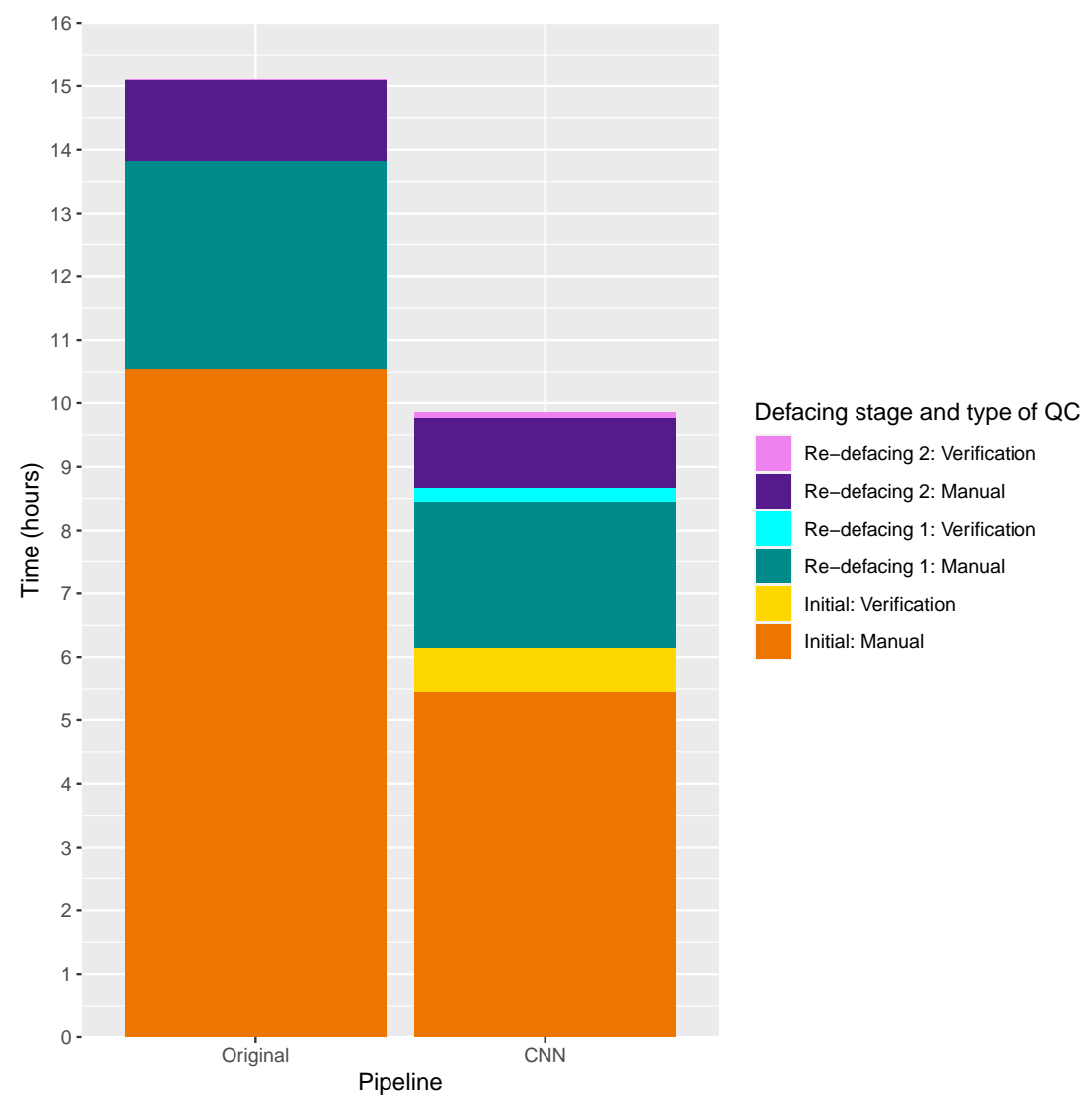

Figure 10: Stacked bar plot comparing the amount of time spent performing QC checks for an example study containing 10,000 scans, using the original fully manual pipeline and the pipeline with the CNN included.

pipeline would have taken 9.8 hours, which is a $35 \%$ reduction in the time spent performing the checks.

\section{Discussion}

In this paper, we were able to develop a CNN that is able to perform QC checks on MRI defacing. Not only can the CNN identify scans that have been incorrectly defaced, it can also distinguish between the type of failure, which can assist when re-defacing these scans. We have shown how the CNN can be integrated within a defacing pipeline in a manner that delivers high standards of QC that protect the identity of subjects. Implementation of the CNN into the pipeline leads to a considerable reduction in the amount of time needed for manual QC checks, leading to faster data turnaround and an increase in capacity for MRI data anonymisation work.

The improved performance of the final model, which was built from scratch, over the transfer learning model, is consistent with findings from similar studies where neural networks have been used to analyse medical imaging datasets (Raghu et al., 2019). Prior to applying probability thresholds to the final CNN, the network delivers high test accuracy (0.76). It is worth noting that this is similar to the performance of models applied to perform other forms of QC on large, multi-site MRI datasets (e.g. Pizarro et al., 2016; Esteban et al., 2017). However, as the CNN is used to QC check patient anonymity, higher levels of accuracy were required. The application of the strict probability thresholds conferred much higher test accuracy (0.92) but still allowed for nearly half of the MRI scans to be QC checked without the 
need for time-consuming manual checks. While it may be possible to further improve the performance of the CNN, allowing for greater proportions of the data to be classified, it seems highly likely that manual QC checks will always be needed for scans that the CNN is unable to reliably classify. Classifying scans into the four discrete categories is not always straightforward, both for humans and the CNN, as scans often exhibit features characteristic of multiple classifications. For example, the front of the brain is often visible through the forehead in correctly defaced scans, or subjects with deeply-set eyes may show features of deep and shallow classifications. Furthermore, scans are often borderline between two categories (typically pass/deep or pass/shallow). In these cases, the CNN can be ambiguous with regard to assigning two (or more) classes. Taking all of this into account, there may be a ceiling for the accuracy of machine learning models that are applied to defacing QC problems.

Once the CNN had been integrated into the pipeline with the selected probability thresholds, nearly half of the scans were classified by the CNN and did not require manual QC checks. The aim of this paper was to develop a model that would assist with the QC of the defacing, and complement, but not replace visual QC checks, while maintaining high QC standards. This goal has been achieved with a reduction in the time spent undertaking manual checks by approximately $35 \%$ while maintaining the quality of the images that are passed through to the analytical pipelines. Further time savings could be found through improving overall model performance, as mentioned above, or by potentially relaxing the probability thresholds. If less strict thresholds were applied, a greater volume of scans could be classified. However, even with the strict thresholds we applied here, up to $10 \%$ of assigned 'pass' classifications were not agreed with during verification checks. Further relaxing of the thresholds would most likely lead to a greater proportion of 'pass' assigned scans needing to be flagged for manual checks, increasing the average time to verify each scan, while making the verification checks less efficient and potentially eroding the time savings that the CNN provides. Furthermore, if the probability thresholds for the 'deep', 'failure', and 'shallow' categories were lowered then the quality of re-defacing attempts could be reduced. During the re-defacing, the custom parameters that are applied are highly dependent on the previous QC classifications. Inaccurate 'failure', 'deep' and 'shallow' classifications would lead to incorrectly applied re-defacing parameters, and a greater proportion of scans not passing the re-defacing, and consequently less data would be available for downstream analysis.

Neural networks are often regarded as 'black box' methods, but with the application of visualisation methods like Grad-CAM we are able to show that our CNN has been trained to rely on the same anatomical features that human scorers use when deciding whether a scan has been defaced correctly. More precisely, we can verify that the network focuses on the eyes, ears, nose and to a lesser extent the mouth regions (defacing issues around the mouth region are less common). In particular, there was strong activation around the eyes for all classes, which is consistent with observations during the manual QC checks - that the eyes are one of the most common areas to exhibit problems. Also, there are rarely issues with the removal of the ears during the defacing, but it is reassuring that despite their rarity, the CNN does seem to be 'seeing' features around this area of the head. Additionally, the CNN is able to correctly classify scans in the registration failure category despite their appearance being quite variable.

For projects like the Novartis-Oxford collaboration, that utilise hundreds of thousands of MRI scans 
acquired from sites around the world, one of the main challenges is being able to process the large volumes of data in a timely manner without compromising on the quality of the processing. While machine learning approaches, such as SVMs and CNNs, are regularly used when performing QC checks on MRI data, they are typically used to identify scans that are likely to be problematic when analysed (e.g. poor image quality, identification of artefacts). Our innovative approach of applying a CNN to assist with QC checking the anonymisation of image data highlights that there is potential for applying machine learning approaches to other stages of MRI processing pipelines. Manual QC checks can be time-consuming bottlenecks, but with the application of approaches such as CNNs, this can be alleviated, expediting the availability of anonymised MRI data to researchers.

\section{Acknowledgements}

The project, and the collaboration, was made possible through access to MRI data from Novartis' MS clinical trials. We wish to thank Piet Arden, Frank Dahlke, and Karine Lheritier from Novartis for their assistance with providing access to data and supporting the collaboration. We wish to acknowledge the help of Mark Jenkinson in providing guidance with establishing the defacing pipeline; Stephen Gardiner, Ewan Straiton, and other members of the data wrangling team for their assistance in working with the MS MRI data; Anna Zalevski and Joanna Stoneham for project management; Adam Huffman, Geoffrey Ferrari, and Robert Esnouf for their work on the IT infrastructure and data transfers for the project.

\section{Funding sources}

This paper is the output from the research collaboration between Novartis and the University of Oxford's Big Data Institute. This study was funded by Novartis, and it uses data collected from Novartis funded clinical trials.

\section{Data statement}

Due to privacy requirements, we are unable to make the underlying data available for sharing.

\section{References}

Abramian, D., Eklund, A., 2019. Refacing: reconstructing anonymized facial features using GANs, in: IEEE 16th International Symposium on Biomedical Imaging (ISBI 2019), pp. 1104-1108. URL: http://arxiv.org/abs/1810.06455, doi:10.1109/ISBI.2019.8759515. arXiv: 1810.06455.

Alfaro-Almagro, F., Jenkinson, M., Bangerter, N.K., Andersson, J.L., Griffanti, L., Douaud, G., Sotiropoulos, S.N., Jbabdi, S., Hernandez-Fernandez, M., Vallee, E., Vidaurre, D., Webster, M., McCarthy, P., Rorden, C., Daducci, A., Alexander, D.C., Zhang, H., Dragonu, I., Matthews, P.M., Miller, K.L., Smith, S.M., 2018. Image processing and Quality Control for the first 10,000 brain imaging datasets from UK Biobank. NeuroImage 166, 400-424. URL: https://linkinghub.elsevier.com/retrieve/pii/S1053811917308613, doi:10.1016/j.neuroimage.2017.10.034. 
bioRxiv preprint doi: https://doi.org/10.1101/2021.06.24.449613; this version posted June 25, 2021. The copyright holder for this preprint (which was not certified by peer review) is the author/funder, who has granted bioRxiv a license to display the preprint in perpetuity. It is made available under aCC-BY-NC-ND 4.0 International license.

Allaire, J.J., Chollet, F., 2018. keras: R interface to 'Keras'. URL: https://CRAN.R-project.org/package=keras.

Bhalerao, G.V., Parekh, P., Saini, J., Venkatasubramanian, G., John, J.P., Viswanath, B., Rao, N.P., Narayanaswamy, J.C., Sivakumar, P.T., Kandasamy, A., Kesavan, M., Mehta, U.M., Mukherjee, O., Purushottam, M., Kannan, R., Mehta, B., Kandavel, T., Binukumar, B., Jayarajan, D., Shyamsundar, A., Moirangthem, S., Vijay Kumar, K., Mahadevan, J., Holla, B., Thirthalli, J., Chandra, P.S., Gangadhar, B.N., Murthy, P., Panicker, M.M., Bhalla, U.S., Chattarji, S., Benegal, V., Varghese, M., Reddy, J.Y., Raghu, P., Rao, M., Jain, S., 2021. Systematic evaluation of the impact of defacing on quality and volumetric assessments on T1-weighted MR-images. Journal of Neuroradiology , S0150986121000559URL: https://linkinghub.elsevier.com/retrieve/pii/S0150986121000559, doi:10.1016/j.neurad.2021.03.001.

Bischoff-Grethe, A., Ozyurt, I.B., Busa, E., Quinn, B.T., Fennema-Notestine, C., Clark, C.P., Morris, S., Bondi, M.W., Jernigan, T.L., Dale, A.M., Brown, G.G., Fischl, B., 2007. A technique for the deidentification of structural brain MR images. Human Brain Mapping 28, 892-903. URL: http://doi.wiley.com/10.1002/hbm.20312, doi:10.1002/hbm.20312.

Chollet, F., Allaire, J.J., 2018. Deep learning with R. Manning, Shelter Island, NY. OCLC: 1022850710.

Dahlke, F., Arnold, D.L., Aarden, P., Ganjgahi, H., Häring, D.A., Čuklina, J., Nichols, T.E., Gardiner, S., Bermel, R., Wiendl, H., 2021. Characterisation of MS phenotypes across the age span using a novel data set integrating 34 clinical trials (NO.MS cohort): Age is a key contributor to presentation. Multiple Sclerosis Journal , 135245852098863URL: http://journals.sagepub.com/doi/10.1177/1352458520988637, doi:10.1177/1352458520988637.

Esteban, O., Birman, D., Schaer, M., Koyejo, O.O., Poldrack, R.A., Gorgolewski, K.J., 2017. MRIQC: Advancing the automatic prediction of image quality in MRI from unseen sites. PLoS ONE 12, e0184661. doi:10.1371/journal.pone.0184661.

Fennema-Notestine, C., Ozyurt, I.B., Clark, C.P., Morris, S., Bischoff-Grethe, A., Bondi, M.W., Jernigan, T.L., Fischl, B., Segonne, F., Shattuck, D.W., Leahy, R.M., Rex, D.E., Toga, A.W., Zou, K.H., Morphometry BIRN, Brown, G.G., 2006. Quantitative evaluation of automated skull-stripping methods applied to contemporary and legacy images: Effects of diagnosis, bias correction, and slice location. Human Brain Mapping 27, 99-113. URL: http://doi.wiley.com/10.1002/hbm.20161, doi:10.1002/hbm.20161.

Gorgolewski, K.J., Auer, T., Calhoun, V.D., Craddock, R.C., Das, S., Duff, E.P., Flandin, G., Ghosh, S.S., Glatard, T., Halchenko, Y.O., Handwerker, D.A., Hanke, M., Keator, D., Li, X., Michael, Z., Maumet, C., Nichols, B.N., Nichols, T.E., Pellman, J., Poline, J.B., Rokem, A., Schaefer, G., Sochat, V., Triplett, W., Turner, J.A., Varoquaux, G., Poldrack, R.A., 2016. The brain imaging data structure, a format for organizing and describing outputs of neuroimaging experiments. Scientific Data 3, 160044. URL: http://www. nature.com/articles/sdata201644, doi:10.1038/sdata.2016.44. 
bioRxiv preprint doi: https://doi.org/10.1101/2021.06.24.449613: this version posted June 25, 2021. The copyright holder for this preprint (which was not certified by peer review) is the author/funder, who has granted bioRxiv a license to display the preprint in perpetuity. It is made available under aCC-BY-NC-ND 4.0 International license.

Gulban, O.F., Nielson, D., Poldrack, R., Lee, J., Gorgolewski, C., Vanessasaurus, Ghosh, S., 2019. poldracklab/pydeface.

Halchenko, Y., Goncalves, M., Castello, V.d.O., Ghosh, S., Hanke, M., Brett, M., Carlin, J., 2019. nipy/heudiconv: Heudiconv. URL: https://github.com/nipy/heudiconv.

Hand, D.J., Till, R.J., 2001. A Simple Generalisation of the Area Under the ROC Curve for Multiple Class Classification Problems. Machine Learning 45, 171-186. doi:10.1023/A:1010920819831.

I Do Imaging, 2017. I Do Imaging. URL: https ://wiki .idoimaging.com/index •php?title=Sample_Data.

Keshavan, A., Yeatman, J.D., Rokem, A., 2019. Combining Citizen Science and Deep Learning to Amplify Expertise in Neuroimaging. Frontiers in Neuroinformatics 13. doi:10.3389/fninf.2019.00029.

Klapwijk, E.T., van de Kamp, F., van der Meulen, M., Peters, S., Wierenga, L.M., 2019. QoalaT: A supervised-learning tool for quality control of FreeSurfer segmented MRI data. NeuroImage 189, 116-129. URL: https://linkinghub.elsevier.com/retrieve/pii/S1053811919300138, doi:10.1016/j.neuroimage.2019.01.014.

Litjens, G., Kooi, T., Bejnordi, B.E., Setio, A.A.A., Ciompi, F., Ghafoorian, M., van der Laak, J.A., van Ginneken, B., Sánchez, C.I., 2017. A survey on deep learning in medical image analysis. Medical Image Analysis 42, 60-88. URL: https://linkinghub.elsevier.com/retrieve/pii/S1361841517301135, doi:10.1016/j.media.2017.07.005.

Mallon, A.M., Häring, D.A., Dahlke, F., Aarden, P., Afyouni, S., Delbarre, D., El Emam, K., Ganjgahi, H., Gardiner, S., Kwok, C.H., West, D.M., Straiton, E., Haemmerle, S., Huffman, A., Hofmann, T., Kelly, L.J., Krusche, P., Laramee, M.C., Lheritier, K., Ligozio, G., Readie, A., Santos, L., Nichols, T.E., Branson, J., Holmes, C., 2021. Advancing data science in drug development through an innovative computational framework for data sharing and statistical analysis. MedRxiv URL: http://medrxiv.org/lookup/doi/10.1101/2021.02.16.21251799, doi:10.1101/2021.02.16.21251799.

Marcus, D.S., Harms, M.P., Snyder, A.Z., Jenkinson, M., Wilson, J.A., Glasser, M.F., Barch, D.M., Archie, K.A., Burgess, G.C., Ramaratnam, M., Hodge, M., Horton, W., Herrick, R., Olsen, T., McKay, M., House, M., Hileman, M., Reid, E., Harwell, J., Schindler, J., Elam, J.S., Curtiss, S.W., Van Essen, D.C., Consortium, W.M.H., 2013. Human Connectome Project Informatics: quality control, database services, and data visualization. NeuroImage 80, 202-219. doi:10.1016/j.neuroimage.2013.05.077.

Mazura, J.C., Juluru, K., Chen, J.J., Morgan, T.A., John, M., Siegel, E.L., 2012. Facial Recognition Software Success Rates for the Identification of 3D Surface Reconstructed Facial Images: Implications for Patient Privacy and Security. Journal of Digital Imaging 25, 347-351. URL: http://link. springer. com/10.1007/s10278-011-9429-3, doi:10.1007/s10278-011-9429-3.

Milchenko, M., Marcus, D., 2013. Obscuring Surface Anatomy in Volumetric Imaging Data. Neuroinformatics 11, 65-75. URL: http://link.springer.com/10.1007/s12021-012-9160-3, doi:10.1007/s12021-012-9160-3. 
bioRxiv preprint doi: https://doi.org/10.1101/2021.06.24.449613: this version posted June 25, 2021. The copyright holder for this preprint (which was not certified by peer review) is the author/funder, who has granted bioRxiv a license to display the preprint in perpetuity. It is made available under aCC-BY-NC-ND 4.0 International license.

Mortamet, B., Bernstein, M.A., Jack, C.R., Gunter, J.L., Ward, C., Britson, P.J., Meuli, R., Thiran, J.P., Krueger, G., 2009. Automatic quality assessment in structural brain magnetic resonance imaging: Automatic QA in Structural Brain MRI. Magnetic Resonance in Medicine 62, 365-372. URL: http://doi.wiley.com/10.1002/mrm.21992, doi:10.1002/mrm.21992.

Pizarro, R.A., Cheng, X., Barnett, A., Lemaitre, H., Verchinski, B.A., Goldman, A.L., Xiao, E., Luo, Q., Berman, K.F., Callicott, J.H., Weinberger, D.R., Mattay, V.S., 2016. Automated Quality Assessment of Structural Magnetic Resonance Brain Images Based on a Supervised Machine Learning Algorithm. Frontiers in Neuroinformatics 10. URL: http://journal.frontiersin.org/article/10.3389/fninf.2016.00052/full, doi:10.3389/fninf.2016.00052.

Prior, F.W., Brunsden, B., Hildebolt, C., Nolan, T.S., Pringle, M., Vaishnavi, S.N., Larson-Prior, L.J., 2009. Facial Recognition From Volume-Rendered Magnetic Resonance Imaging Data. IEEE Transactions on Information Technology in Biomedicine 13, 5-9. doi:10.1109/TITB.2008.2003335.

R Core Team, 2018. R: A language and environment for statistical computing. URL: https://www.R-project.org.

Raghu, M., Kleinberg, J., Zhang, C., Bengio, S., 2019. Transfusion: Understanding Transfer Learning for Medical Imaging. arXiv, 11.

Reuter, M., Tisdall, M.D., Qureshi, A., Buckner, R.L., van der Kouwe, A.J., Fischl, B., 2015. Head motion during MRI acquisition reduces gray matter volume and thickness estimates. NeuroImage 107, 107-115. URL: https://linkinghub.elsevier.com/retrieve/pii/S1053811914009975, doi:10.1016/j.neuroimage.2014.12.006.

Robin, X., Turck, N., Hainard, A., Tiberti, N., Lisacek, F., Sanchez, J.C., Müller, M., 2011. pROC: an open-source package for $\mathrm{R}$ and $\mathrm{S}+$ to analyze and compare ROC curves. BMC Bioinformatics 12 , 77. URL: https://bmcbioinformatics .biomedcentral . com/articles/10.1186/1471-2105-12-77, doi:10.1186/1471-2105-12-77.

Schimke, N., Hale, J., 2011. Quickshear Defacing for Neuroimages, in: HealthSec'11: Proceedings of the 2nd USENIX Conference on Health Security and Privacy, p. 11.

Schimke, N., Kuehler, M., Hale, J., 2011. Preserving Privacy in Structural Neuroimages, in: Hutchison, D., Kanade, T., Kittler, J., Kleinberg, J.M., Mattern, F., Mitchell, J.C., Naor, M., Nierstrasz, O., Pandu Rangan, C., Steffen, B., Sudan, M., Terzopoulos, D., Tygar, D., Vardi, M.Y., Weikum, G., Li, Y. (Eds.), Data and Applications Security and Privacy XXV. Springer Berlin Heidelberg, Berlin, Heidelberg. volume 6818, pp. 301-308. URL: http://link. springer .com/10.1007/978-3-642-22348-8_26, doi:10.1007/978-3-642-22348-8_26. series Title: Lecture Notes in Computer Science.

Schwarz, C.G., Kremers, W.K., Therneau, T.M., Sharp, R.R., Gunter, J.L., Vemuri, P., Arani, A., Spychalla, A.J., Kantarci, K., Knopman, D.S., Petersen, R.C., Jack, C.R., 2019. Identification of 
bioRxiv preprint doi: https:/doi.org/10.1101/2021.06 24.449613. this version posted June 25,2021 . The copyright holder for this preprint (which was not certified by peer review) is the author/funder, who has granted bioRxiv a license to display the preprint in perpetuity. It is made available under aCC-BY-NC-ND 4.0 International license.

Anonymous MRI Research Participants with Face-Recognition Software. The New England Journal of Medicine , 1684-1686doi:10.1056/NEJMc1908881.

Schwarz, C.G., Kremers, W.K., Wiste, H.J., Gunter, J.L., Vemuri, P., Spychalla, A.J., Kantarci, K., Schultz, A.P., Sperling, R.A., Knopman, D.S., Petersen, R.C., Jack, C.R., 2021. Changing the face of neuroimaging research: Comparing a new MRI de-facing technique with popular alternatives. NeuroImage 231, 117845. URL: https://linkinghub.elsevier.com/retrieve/pii/S1053811921001221, doi:10.1016/j.neuroimage.2021.117845.

Ségonne, F., Dale, A., Busa, E., Glessner, M., Salat, D., Hahn, H., Fischl, B., 2004. A hybrid approach to the skull stripping problem in MRI. NeuroImage 22, 1060-1075. URL: https://linkinghub.elsevier.com/retrieve/pii/S1053811904001880, doi:10.1016/j.neuroimage.2004.03.032.

Selvaraju, R.R., Cogswell, M., Das, A., Vedantam, R., Parikh, D., Batra, D., 2017. GradCAM: Visual Explanations from Deep Networks via Gradient-Based Localization, in: 2017 IEEE International Conference on Computer Vision (ICCV), IEEE, Venice. pp. 618-626. URL: http://ieeexplore.ieee.org/document/8237336/, doi:10.1109/ICCV.2017.74.

Shattuck, D.W., Sandor-Leahy, S.R., Schaper, K.A., Rottenberg, D.A., Leahy, R.M., 2001. Magnetic Resonance Image Tissue Classification Using a Partial Volume Model. NeuroImage 13, 856-876. URL: https://linkinghub.elsevier.com/retrieve/pii/S1053811900907304, doi:10.1006/nimg.2000.0730.

Smith, S.M., 2002. Fast robust automated brain extraction. Human Brain Mapping 17, 143-155. URL: http://doi.wiley.com/10.1002/hbm.10062, doi:10.1002/hbm.10062.

Theyers, A.E., Zamyadi, M., O'Reilly, M., Bartha, R., Symons, S., MacQueen, G.M., Hassel, S., Lerch, J.P., Anagnostou, E., Lam, R.W., Frey, B.N., Milev, R., Müller, D.J., Kennedy, S.H., Scott, C.J.M., Strother, S.C., 2021. Multisite Comparison of MRI Defacing Software Across Multiple Cohorts. Frontiers in Psychiatry 12, 617997. URL: https://www frontiersin.org/articles/10.3389/fpsyt.2021.617997/full, doi:10.3389/fpsyt.2021.617997. 